\title{
Ultrasonography and low-field magnetic resonance imaging of the common calcanean tendon in a rabbit model for tendinopathy research: a descriptive study of normal anatomy
}

\author{
A. Skalec ${ }^{1}$, P. Przyborowska-Zhalniarovich², I. Janus ${ }^{3}$, K. Kirstein ${ }^{1}$, \\ M. Mieszkowska², Z. Adamiak², A. Chrószcz ${ }^{1}$, M. Janeczek ${ }^{1}$ \\ ${ }^{1}$ Department of Animal Physiology and Biostructure, Faculty of Veterinary Medicine, \\ Wroclaw University of Environmental and Life Sciences, Kożuchowska 1/3, 51-631 Wrocław, Poland \\ ${ }^{2}$ Department of Surgery and Radiology, Faculty of Veterinary Medicine, \\ University of Warmia and Mazury, Olsztyn, Poland \\ ${ }^{3}$ Department of Pathology, Faculty of Veterinary Medicine, \\ Wroclaw University of Environmental and Life Sciences, Poland
}

\begin{abstract}
In spite of recent advances in treatment protocols, tendinopathies continue to challenge orthopaedists and surgeons. Due to the complexity of both tendon injuries and the healing processes, animal models are essential for addressing fundamental questions in tendinopathy research. Diagnostic imaging could contribute to the evaluation of animal models, thus providing information, which could be translated to human tendinopathies. The objective of our study was to evaluate in situ appearance of the rabbit common calcanean tendon with ultrasonography and magnetic resonance imaging. Additionally, we sought to assess and compare the feasibility and usefulness of these techniques in a rabbit model while focusing on the imaging of the particular structures involved in calcaneal tendon disorders. Eight California rabbits were used for post-mortem sonographic and low-field magnetic resonance examination. Morphometry was performed on longitudinal sonograms and sagittal MRI scans. The craniocaudal diameter of the tendon was measured at four points of interest. Ultrasonography and magnetic resonance provided good visualisation of the tendon origin, the paratenon and the pre-Achilles fat pad. Magnetic resonance images presented in more detail the structure of the calcaneal insertion. Both modalities failed to visualise the individual components of the common calcanean tendon and the bursa of the calcaneal tendon. Statistical analysis of measurements obtained showed that the craniocaudal diameter of the common calcanean tendon in a rabbit increases significantly with a growing length from the calcaneal tuber. Both magnetic resonance and ultrasonography are feasible, and should be considered complementary, not alternative imaging techniques in a rabbit common calcanean tendon model.
\end{abstract}

Key words: animal model, rabbit, anatomy, MRI, ultrasonography, common calcanean tendon 


\section{Introduction}

Tendon diseases are common and often devastating injuries among humans and might lead to significant disability, exercise associated pain and decreased functionality (Thomopoulos et al. 2015). Despite recent advances in the treatment protocols, their outcomes are still unpredictable due to the decreased mechanical properties of fibrovascular scarring and the degenerative nature of many tendon disorders (Kader et al. 2002, Shapiro et al. 2015). It has been stated that the calcaneal tendon (Achilles tendon) is one of the most frequently injured tendons in the human musculoskeletal system (Pierre-Jerome et al. 2010, Del Buono et al. 2013). Due to the complexity of tendon injury and the healing processes as well as differences among particular tendinopathic conditions, animal models are essential for addressing fundamental questions in tendon research (Thomopoulos et al. 2015). Experimental models used to investigate various treatment protocols are usually evaluated by means of histopathological, biochemical or mechanical examinations. However, in practice, diagnostic imaging along with clinical examination are commonly applied for the calcaneal tendon assessment (Bleakney and White 2005, Harris and Peduto 2006, Pierre-Jerome et al. 2010, Del Buono et al. 2013). Ultrasonography and magnetic resonance imaging provide a non-invasive assessment of tendons, allowing on defining stage of the extend and severity of the disease and evaluation of applied treatment effectiveness. Diagnostic imaging could contribute greatly to the evaluation of animal models while providing additional information which could be further translated to the clinical picture of human tendinopathies. Rabbits are commonly used in experimental studies focused on Achilles tendon pathologies and their repair (Hast et al. 2014, Thomopoulos et al. 2015). However, it should be emphasized that there are some limitations concerning experimental protocols resulting from differences in anatomy of the pelvic limb between quadruped laboratory animals and biped humans. According to Nomina Anatomica Veterinaria, the common calcanean tendon is a convenient designation for the aggregated tendons in the distal part of the crus which are attached to the calcaneal tuber in domestic mammals. It includes the tendons of the superficial digital flexor, the triceps surae and a tendinous contribution from the biceps femoris and the semitendinosus. The triceps surae is composed of the gastrocnemius, with its lateral and medial head, and soleus, which is only negligible in a rabbit (Doherty et al. 2006). Conjoint tendons of the triceps surae create the calcaneal tendon (Achilles tendon). The most important difference in anatomy of this region between humans and quadruped mammals is the lack of the superficial digital flexor in the former. Consequently, we do not distinguish the common calcanean tendon in a man and all described Achilles tendinopathies refer clearly to the calcaneal tendon, i.e. the tendons of the gastrocnemius and soleus (Szaro et al. 2009, Doral et al. 2010). It should be kept in mind that, the analysis of the experimental research with laboratory animals utilizing the components of the common calcanean tendon, like superficial digital flexor tendon or tendons of the medial or lateral head of the gastrocnemius, are applied to the human calcaneal tendon pathologies only by analogy and not by straightforward translation. Experimental study design should also include the evaluation of anatomical structures which are commonly involved in Achilles tendon related disorders in humans (van Dijk et al. 2011). The Kager's (pre-Achilles) fat pad is a lipomatous structure located posterior to the talocalcaneal joint and anterior to the Achilles tendon in a standing man (Ly et al. 2004, Theobald et al. 2006, Benjamin et al. 2008). The pre-Achilles fat pad is considered as a mechanosensory organ for the calcaneal tendon and is implicated in tendinopathies (Benjamin et al. 2008). It also minimizes pressure changes in the bursa of the calcaneal tendon, plays a role in a tendon biomechanics and protects the blood vessels supplying the calcaneal tendon (Theobald et al. 2006). There is no specific nomenclature for analogical fat pad present in a rabbit and for this reason authors will use the term pre-Achilles fat pad for the purpose of this paper. The bursa of the calcaneal tendon (in humans also called the retrocalcaneal bursa) can also be affected, especially accompanying the insertional tendinopathy of the calcaneal tendon (van Dijk et al. 2011). The inflammation of this bursa is referred to as the retrocalcaneal bursitis and can be identified as a fluid accumulation in ultrasonography (hyperechoic area) or as a hyperintense signal in magnetic resonance imaging (van Dijk et al. 2011). This synovial bursa is also present in domestic mammals sharing the same name and located between the calcaneal tuber and the common calcanean tendon. According to the contribution of the bursa of calcaneal tendon in Achilles tendinopathies it should also be evaluated in experimental research using animal models. The pre-Achilles fat pad, the bursa of the calcaneal tendon, the calcaneal tendon enthesis itself as well as the fibrocartilages on the surface of the tendon and on the opposing calcaneal tuberosity, formed as a result of an intermittent contact between the tendon and bone, constitute the enthesis organ affected in the spondyloarthropathies of the Achilles tendon (Benjamin and McGonagle 2009). Additionally, the calcaneal tendon is an example of a tendon surrounded 
by the paratenon - a double-layered connective tissue membrane, separated from the tendon itself (Benjamin et al. 2008, Pierre-Jerome et al. 2010). It is involved in Achilles tendon pathologies, classified as paratendinopathies (van Dijk et al. 2011). Experimental studies in which the diagnostic imaging is used as an assessment tool to evaluate obtained results should include the structures presented above in correlation to technical capabilities of such examination. The rabbit common calcanean tendon is relatively large in comparison to that in other small laboratory animals, which makes it more accessible for diagnostic imaging. Previously, we have described the ultrasonographic anatomy and morphometry of the rabbit common calcanean tendon (Skalec et al. 2016), but there is a lack of a detailed description of its in situ appearance in magnetic resonance imaging.

The aim of the present study was to qualitatively and quantitatively compare ultrasonography and low-field magnetic resonance imaging in the assessment of the normal anatomy of the common calcanean tendon in a rabbit model. Additionally, we aimed to describe the baseline appearance of the tendon and its related structures in these imaging modalities.

\section{Materials and Methods}

Eight skeletally-mature California rabbits (6 males and 2 females) of a mean body weight of $5.92 \mathrm{~kg}(5.54$ $\mathrm{kg}-6.39 \mathrm{~kg}$ ) were used in the study (Rabbit Farm 183-P Marcin Kaletka, Wielichowo, Poland). All the animals were euthanized prior to the examination utilizing an intravenous pentobarbital injection (200 $\mathrm{mg} / \mathrm{kg}$ ) in the course of a different experiment not related to this study (approval by II Local Ethics Committee of Wroclaw University of Environmental and Life Sciences, Poland, decision No. 100/2014 of 17/09/2014). Post-mortem ultrasonographic and magnetic resonance imaging of the common calcanean tendons of both pelvic limbs in each rabbit was performed within 48 hours after euthanasia. Ultrasonography was carried out immediately after sacrificing the animals. The cadavers were not frozen subsequently, but stored in a temperature-controlled cool room for the magnetic resonance imaging and were allowed to gain room temperature before scanning. This protocol was design according to guidelines for postmortem MRI of musculoskeletal structures (van der Made et al. 2013).

Sonographic examination was performed using a Sonoscape $\mathrm{S} 6 \mathrm{~V}^{\circledR}$ unit with a linear-array transducer featuring a frequency range of 5-13 $\mathrm{MHz}$ (Sonomedical, Poznań, Poland). The skin over both common cal- canean tendons was shaved, moistened with surgical spirit and covered with ultrasound coupling gel. The animals were positioned in lateral recumbency with the examined limb flexed in the tarsal joint at approximately a 90 degree angle, thus assuring adequate tension of the tendon as well as providing good contact between the skin and the ultrasound probe. All images were obtained by one operator in order to avoid interoperator variabilities during image acquisition (A.S.). Tendons were scanned in longitudinal and transverse planes from a caudal approach. The orientation point in the longitudinal projection was the calcaneal tuber.

Low-field magnetic resonance imaging was performed using an Esaote Vet-MR Grande ${ }^{\circledR} 0.25$ Tesla scanner (Med4Animals, V.D. White Med, Warszawa, Poland). The animals were positioned in lateral recumbency with the examined limb on top, flexed in the tarsal joint at approximately 90 degrees and placed in the metacarpus coil. The contralateral pelvic limb was extended backwards. Scans were obtained in transverse and sagittal planes by one operator (P.P.). The protocol consisted of a sagittal T1- weighted spine echo (echo time [TE] $18 \mathrm{~ms}$, repetition time [TR] $600 \mathrm{~ms}$, acquisition matrix $288 * 200$, field of view [FOV] 170*170 mm, slice thickness $3 \mathrm{~mm}$, interslice gap 0mm, number of slices 12, acquisition time 4:03 min:s), sagittal T2-weighted fast spine echo (TE 90 ms, TR $3440 \mathrm{~ms}$, matrix 352*352, FOV 220*220 mm, slice thickness $3 \mathrm{~mm}$, interslice gap $0 \mathrm{~mm}$, number of slices 12, time 5:37 min:s) and transverse T2- weighted fast spine echo sequences (TE $90 \mathrm{~ms}$, TR $4870 \mathrm{~ms}$, matrix $256 * 208$, FOV $160 * 160 \mathrm{~mm}$, slice thickness $3 \mathrm{~mm}$, interslice gap $0 \mathrm{~mm}$, number of slices 17 , time 4:42 min:s). Transverse scans were acquired perpendicular to the long axis of the common calcanean tendon. Sagittal scans were acquired parallel to the median plane of the pelvic limb.

Analysis of images obtained with ultrasonography and scans obtained with magnetic resonance was undertaken by one operator (A.S.). Visualisation of the major components of the common calcanean tendon, that is tendons of the lateral and medial head of the gastrocnemius and the superficial digital flexor tendon (Fig. 1) was evaluated. This protocol was determined not only based on significant contribution of these tendons to the common calcanean tendon but also according to their applicability in tendinopathy research in a rabbit model (Young et al. 1998, Moshiri and Oryan 2011, Oryan and Moshiri 2011, Oryan et al. 2012). Sonograms and scans were assessed qualitatively with a description of normal appearance of elements analogical to those assessed in human Achilles tendon pathologies, that means echogenicity or signal intensity (depending on imaging technique) 


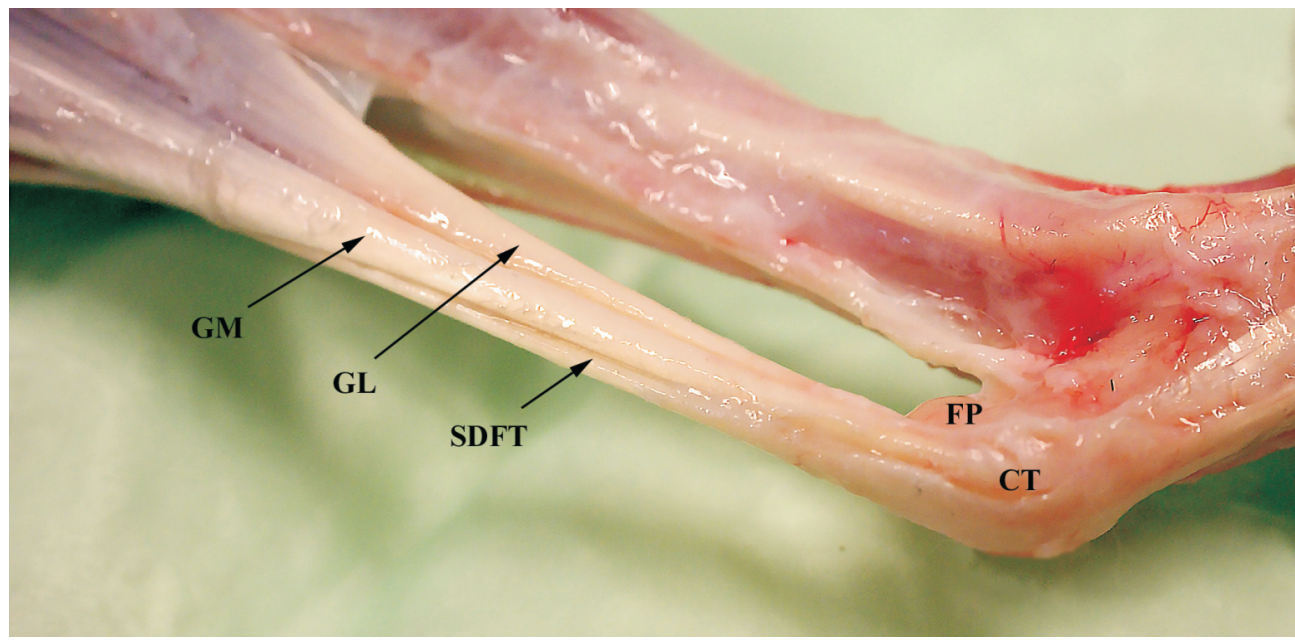

Fig. 1. The rabbit common calcanean tendon at post-mortem dissection - caudolateral view. Presented are three main tendinous components: superficial digital flexor tendon (SDFT), tendons of the medial (GM) and lateral (GL) head of the gastrocnemius. Note the lateral rotation of tendons. CT - calcaneal tuber, FP - pre-Achilles fat pad.

of the tendon, musculotendinous junctions, the region of calcaneal insertion, the paratenon, the pre-Achilles fat pad and the bursa of calcaneal tendon (Kamel et al. 2003, Bleakney and White 2005, Harris and Peduto 2006, Leung and Griffith 2008, McGonagle et al. 2008, Pierre-Jerome et al. 2010, van Dijk et al. 2011, Del Buono et al. 2013). Morphometry of the common calcanean tendon was performed on longitudinal sonograms and sagittal T1-weighted MRI (magnetic resonance imaging) scans presenting median section of the crus. The craniocaudal dimension of the tendon was measured at the level of the calcaneal tuber as well as 1,2 and $3 \mathrm{~cm}$ proximally, with a software dedicated to each imaging unit. Image analysis was performed on stored scans the day after obtaining the images. All measurements were obtained three times, in order to calculate the mean value used for the statistical analysis.

The statistical analysis was performed using StatisticaPL ${ }^{\circledR}$ software (StatSoft Polska, Kraków, Poland). The following tests were used: the Shapiro-Wilk test to determine the normality of the data distribution, the Mann-Whitney $U$ test to determine differences between the results obtained from the male and female rabbits as well as from the left and right limbs, Kruskal-Wallis analysis to determine the differences between the measurements at various distances from the calcaneal tuber, the Mann-Whitney U test to compare the results yielded with sonography and magnetic resonance at every measurement point, and finally Spearman's correlation test to assess the correlation between the variables. The level of statistical significance was set at $\mathrm{p} \leq 0.05$.

\section{Results}

\section{Ultrasonography}

In the longitudinal ultrasonographic images the structure of the common calcanean tendon was clearly visualised. As we reported previously (Skalec et al. 2016), it could be divided, due to echogenicity, into the superficial hypoechoic part and the deeper hyperechoic component (Fig. 2). However, in our previous study, the ultrasound probe was placed slightly to the medial side to assure good visualisation of the superficial digital flexor tendon. In these circumstances the superficial structure corresponds to the superficial digital flexor tendon and the deeper component, with tightly packed thin echogenic lines, to tendons of the lateral and medial head of the gastrocnemius. Placing the probe in the median plane of the common calcanean tendon, the superficial component can correspond to both the superficial digital flexor tendon and the tendon of the medial head of the gastrocnemius. These tendons run side by side for a part of their way to the calcaneal tuber. The direct distinction between the major individual components of the common calcanean tendon was impossible to obtain in the single, static longitudinal plane. The most proximal aspect of the calcaneal tuber was visible as a thin hyperechoic line, but the detailed architecture of the calcaneal insertion was not obtained. The musculotendinous junctions of the gastrocnemius and the superficial digital flexor were well defined, but required dynamic examination. The paratenon was visible as a slightly more echogenic line around the common calcanean tendon. The pre-Achilles fat pad was hypoechogenic or even anechogenic with an irregular 


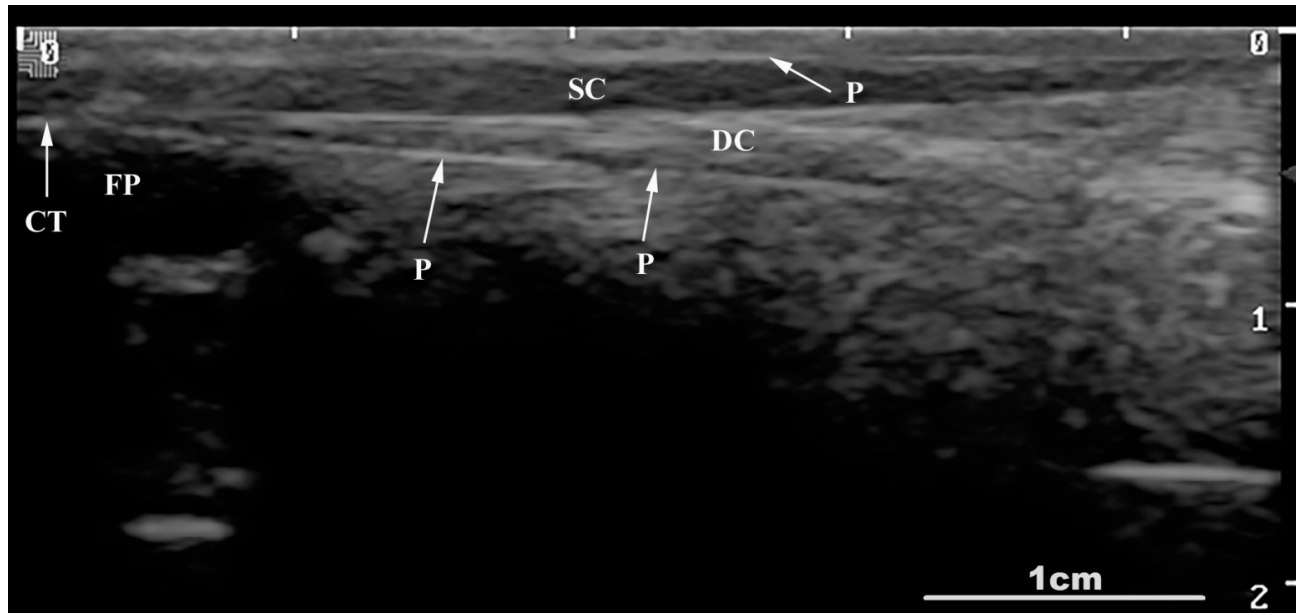

Fig. 2. Longitudinal sonogram of the rabbit common calcanean tendon. Caudal is to the top, proximal is to the right. The superficial (SC) and deep components are clearly distinguishable. The paratenon (P) is well defined. CT - calcaneal tuber, FP - pre-Achilles fat pad.

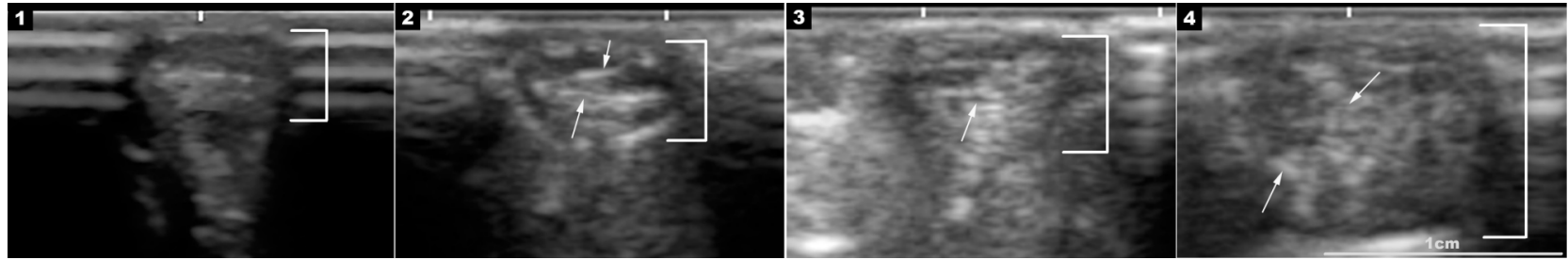

Fig. 3. Transverse sonograms of the rabbit common calcanean tendon directly proximal to the calcaneal tuber (1) and $1 \mathrm{~cm}(2)$, $2 \mathrm{~cm}$ (3) and $3 \mathrm{~cm}$ (4) proximal to the calcaneal tuber. Caudal is to the top, lateral is to the right. Brackets on the right indicate the craniocaudal dimension of the tendon. Cross-sections present varied echogenicity with linear and punctate hyperechogenic foci (arrows).

outline, localised between the cranial aspect of the calcaneal tuber and the common calcanean tendon. In transverse images it was impossible to distinguish particular tendinous components of the common calcanean tendon. The cross-sectional area presented varied echotexture with echogenic punctate foci. Proximally, the common calcanean tendon was round in the transverse section and became slightly ovoid, wider and flattered distally (Fig. 3).

\section{Magnetic resonance imaging}

In all MRI sequences obtained, the common calcanean tendon was of low signal intensity. According to the Chan classification (Del Buono et al. 2013), the calcaneal tendon in humans could be divided into musculotendinous and insertional (calcaneal) portions. Adopting this classification to the rabbit model, the musculotendinous component of the common calcanean tendon consisted of the intramuscular part, corresponding to the tendinous plates of the gastrocnemius and the superficial digital flexor, and the free tendon. In sagittal scans, linear areas of higher signal intensity could be recognised representing the epitenon of particular components. Cranial and caudal margins of the tendon were parallel and concave proximally, i.e. in the intramuscular portion. Distally, at the level of the free tendon, the margins were straight and no longer parallel to one another, but came closer together in the distal direction. At the calcaneal insertion, tendon margins became convex as they passed over the most proximal aspect of the calcaneal tuber. Individual components of the common calcanean tendon could not be clearly identified on a single MRI slice along their overall course to the calcaneal tuber. Musculotendinous junctions of the gastrocnemius and the superficial digital flexor were visualised as areas of changing signal intensity, from a moderate signal of the muscle bellies to a low signal intensity of the tendons. The gastrocnemius musculotendinous junction could be identified at the proximal and the superficial digital flexor's at the most distal end of the intramuscular portion of the common calcanean tendon. Additionally, at the musculotendinous junction of the superficial digital flexor there was a line of high signal intensity between the muscle and tendon. At the level of the calcaneal 


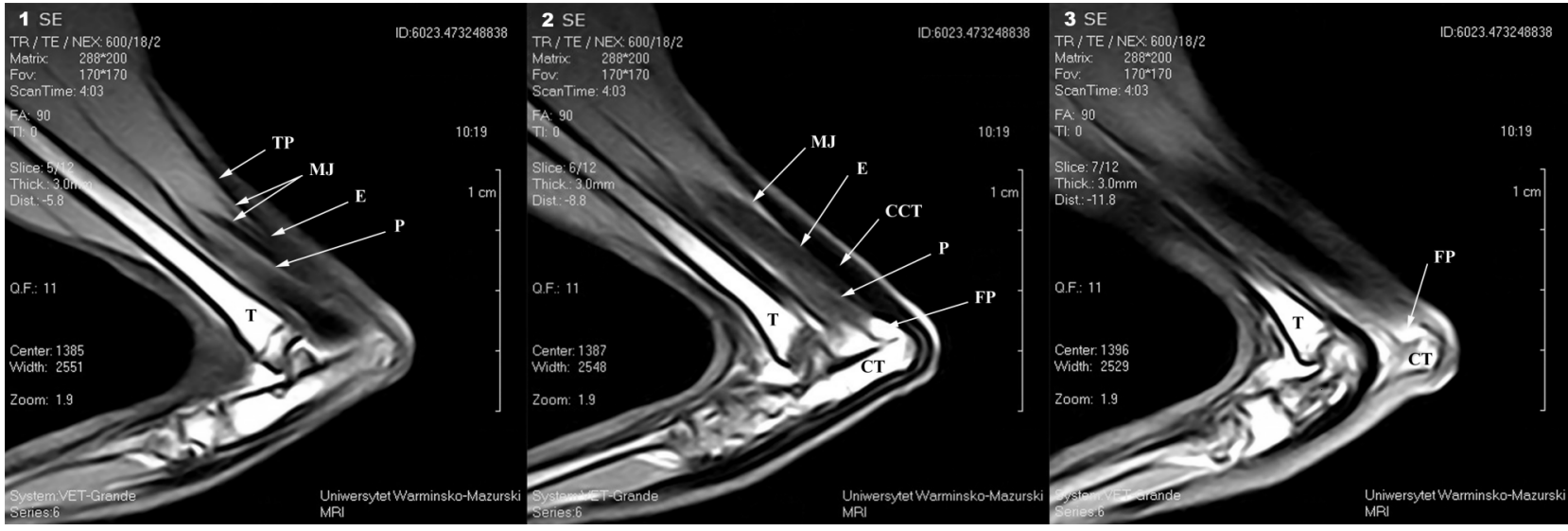

Fig. 4. T1-weighted sagittal MRI scans presenting three following slices. In the median plane of the crus (2) the common calcanean tendon is most visible but due to complex anatomy, more than one slice is needed to fully analyse its structure. CCT - common calcanean tendon, TP - tendinous plate of gastrocnemius, $\mathrm{MJ}$ - musculotendinous junctions, $\mathrm{E}$ - epitenon, $\mathrm{P}$ - paratenon, T - tibia, CT - calcaneal tuber, FP - pre-Achilles fat pad.

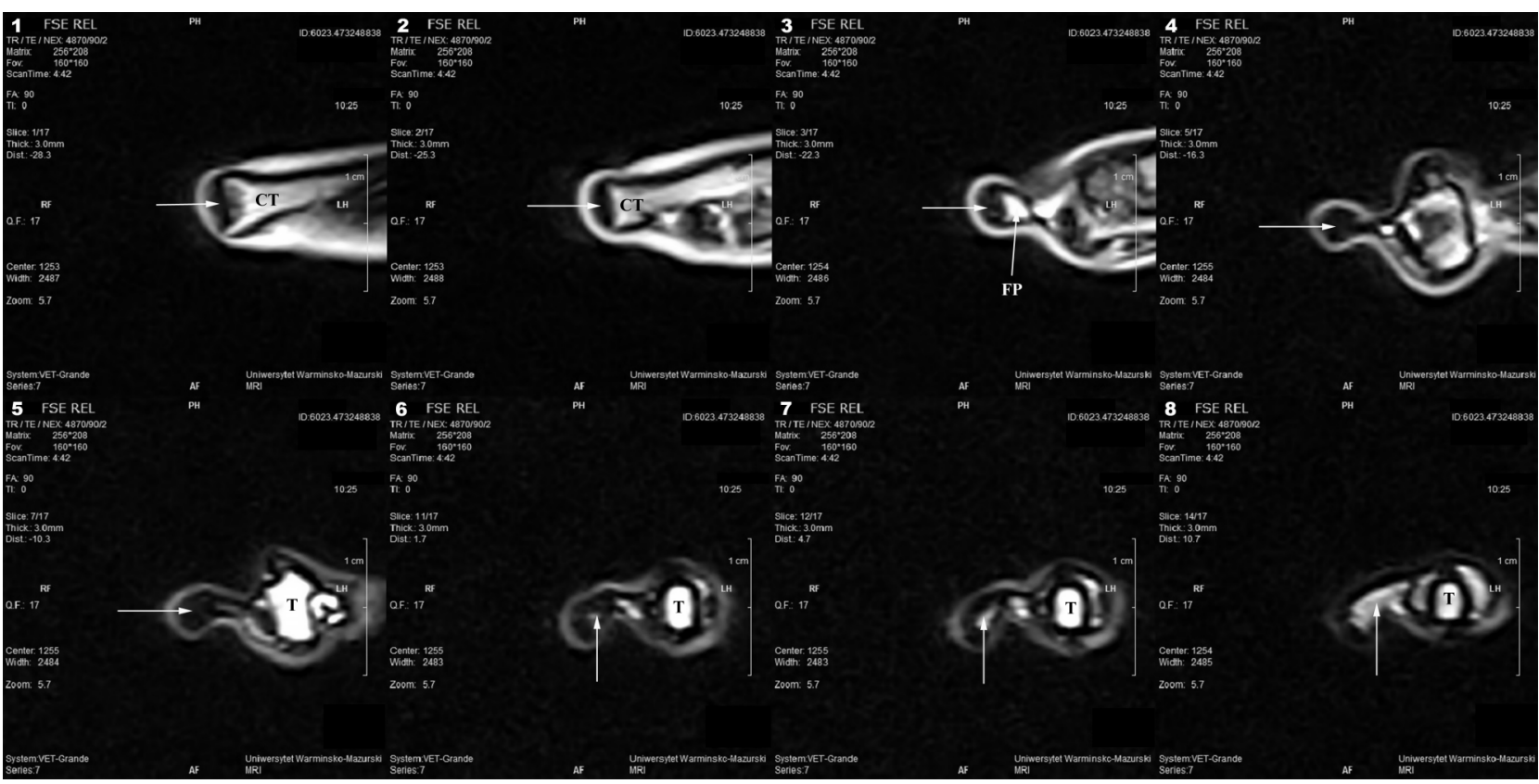

Fig. 5. T2 - weighted transverse MRI scans of the rabbit common calcanean tendon. (1) and (2) level of the calcaneal tuber; (3) $3 \mathrm{~mm}$ proximal; (4) $9 \mathrm{~mm}$; (5) $15 \mathrm{~mm}$; (6) $27 \mathrm{~mm}$; (7) $30 \mathrm{~mm}$ and (8) $36 \mathrm{~mm}$ proximal to the calcaneal tuber (CT). The common calcanean tendon (horizontal arrow) changes its shape from flattened, ovoid to rounded with the growing length from the calcaneal tuber. $3 \mathrm{~mm}$ above CT, the pre-Achilles fat pad (FP) is visible cranially to the tendon. Approximately $30 \mathrm{~mm}$ proximal to CT hyperintensive dot (vertical arrow) indicates the beginning of the musculotendinous junction. T- tibia.

tuber, the superficial digital flexor tendon was easily distinguishable from the fused tendons of the medial and lateral head of the gastrocnemius, these components were separated by a line of higher signal intensity. The calcaneal portion of tendons of both heads of the gastrocnemius and their exact insertion on the calcaneal tuber was clearly visible. The paratenon of the common calcanean tendon was evident cranially as a thin line surrounding the tendon, slightly hyperin- tense to muscles. Caudally it could not be separated from the subcutaneous tissue and the skin. The pre-Achilles fat pad presented high signal intensity. It was of a triangular shape, limited caudally by the common calcanean tendon, distally by the calcaneal tuber and contacting cranially with a connective tissue separating the tendon from the deep musculature of the crus. To thoroughly analyse the anatomy of this region in MRI scans, it was necessary to assess at least 
two sagittal slices due to the lateral rotation of the common calcanean tendon components and their relatively small dimensions in relation to the $3 \mathrm{~mm}$ slice thickness (Fig. 4). In transverse scans, the tendon was also of low signal intensity, but with internal punctate foci of higher intensity. However, there were no visible septa that would clearly divide cross-sections on particular components. The shape of the common calcanean tendon changed through its course. At the insertional portion it was almost flat with convex caudal and cranial margins. At the level of the free tendon, the shape was ovoid and it contacted the widest side of the triangular pre-Achilles fat pad. Above it, the tendon became round, and at the level of the musculotendinous junction of superficial digital flexor it gained a well defined hyperintensive dot in the centre. This area became a muscle belly of moderate signal intensity in the intramuscular portion of the common calcanean tendon, which changed to a narrow and crescent shaped (Fig. 5).

The bursa of the calcaneal tendon was not detected either ultrasonographically or by means of magnetic resonance imaging.

\section{Morphometry and statistical analysis}

Mean values of morphometrical measurements of the craniocaudal diameter of the common calcanean tendon obtained with ultrasonography were $2.50 \mathrm{~mm}$ [standard deviation (SD) 0.29] at the level of the calcaneal tuber, $3.39 \mathrm{~mm}$ (SD 0.39) $1 \mathrm{~cm}$ proximally from the calcaneal tuber, $4.22 \mathrm{~mm}$ (SD 0.4 ) $2 \mathrm{~cm}$ proximally and $5.01 \mathrm{~mm}$ (SD 0.45$) 3 \mathrm{~cm}$ proximally from the calcaneal tuber. Mean values obtained with magnetic resonance imaging were $2.73 \mathrm{~mm}$ (SD 0.31), $3.73 \mathrm{~mm}$ (SD 0.50), $4.48 \mathrm{~mm}$ (SD 0.42) and $5.04 \mathrm{~mm}$ (SD 0.63) in corresponding points of interest. The statistical analysis showed no significant differences in the measurements of the common calcanean tendon between male and female rabbits $(p>0.05)$ or between the contralateral pelvic limbs $(p>0.05)$ obtained with ultrasonography and magnetic resonance. Kruskal-Wallis analysis showed a significant difference in the measurements taken at various distances from the calcaneal tuber with both imaging techniques $(\mathrm{p}<0.001)$. The Mann-Whitney U test showed no statistically significant differences between the sonographic and MRI measurements of the common calcanean tendon at all points of interest, that is: at the level of the calcaneal tuber $(\mathrm{p}=0.56), 1 \mathrm{~cm} \quad(\mathrm{p}=0.28), 2 \mathrm{~cm}$ $(\mathrm{p}=0.16)$ and $3 \mathrm{~cm}$ proximally from the calcaneal tuber $(p=0.9)$. The Spearman test showed a correlation between the ultrasonographic measurements of the common calcanean tendon obtained 1 and $2 \mathrm{~cm}$ proximally from the calcaneal tuber $(\mathrm{p}<0.05, \mathrm{r}=0.65)$ as well as between these obtained at distances of 2 and $3 \mathrm{~cm}(\mathrm{p}<0.05, \mathrm{r}=0.53)$. The positive correlation was also found between measurements of the common calcanean tendon obtained with magnetic resonance for the given points of interest: at the level of the calcaneal tuber and $1 \mathrm{~cm}$ proximally $(\mathrm{p}<0.05, \mathrm{r}=0.65)$, 1 and $2 \mathrm{~cm}(\mathrm{p}<0.05, \mathrm{r}=0.54)$ as well as 2 and $3 \mathrm{~cm}$ proximal to the calcaneal tuber $(\mathrm{p}<0.05, \mathrm{r}=0.54)$. Analysing relations between the results obtained with sonography and magnetic resonance, the Spearman test showed a positive correlation between measurements performed at the point $2 \mathrm{~cm}$ proximal to the calcaneal tuber $(\mathrm{p}<0.05, \mathrm{r}=0.75)$.

\section{Discussion}

The knowledge of the anatomy of the rabbit common calcanean tendon and its appearance in imaging modalities has a significant role in planning experimental research using this model and for generalizing the results to human tendinopathies. In rabbits, the calcaneal tendon is composed of tendons of the lateral and medial head of the gastrocnemius. The soleus gives only negligible contribution to the tendon of the lateral gastrocnemius head. The tendon fibres of both heads of gastrocnemius converge at the level of ca. $93 \%$ of their overall course to the calcaneal tuber (Doherty et al. 2006). In the proximodistal direction the tendon of the medial head of the gastrocnemius is located more caudally than the tendon of the lateral head. The calcaneal tendon is accompanied by the superficial digital flexor tendon and together they form the common calcanean tendon. As these tendons track distally, they rotate in a lateral direction, which reflects the spiral arrangement of the fibers and fascicles of the human Achilles tendon (Doherty et al. 2006, Szaro et al. 2009, Doral et al. 2010). Due to the lateral rotation, at the level of the calcaneal tuber the superficial digital flexor tendon is positioned immediately caudal to the calcaneal tendon insertion (Doherty et al. 2006).

There are few publications which report the use of grey scale ultrasonography in the evaluation of experimental treatment protocols in a rabbit common calcanean tendon model (Young et al. 1998, Moshiri and Oryan 2011, Oryan and Moshiri 2011, Oryan et al. 2012), and we have discussed them previously with our results (Skalec et al. 2016). In a study describing the anatomic relationship between the calcaneal tendon and superficial digital flexor tendon in a rabbit, the authors performed sonography and magnetic resonance imaging (Doherty et al. 2006). In sagittal MRI scans individual tendons were difficult to distinguish. 
Our results confirm this difficulty. However, in transverse scans researchers were able to identify and measure these individual components, but that was not possible in our study. These differences may be the result of a higher magnetic field strength of 1.5 Tesla used in a MRI unit by Doherty et al. (Doherty et al. 2006). In another study, assessing the usefulness of magnetic resonance imaging, ultrasound imaging or measurement of bone mineral density in predicting the mechanical properties of immobilized rabbit calcaneal tendons, the authors were able to measure the cross-sectional area, craniocaudal diameter and T1-signal intensity using a 1.5 Tesla MRI scanner (Trudel et al. 2007). However, in both studies imaging examinations were performed on isolated specimens of tendons resected en bloc and to the best of our knowledge there is no paper describing and comparing the in situ appearance of the rabbit common calcanean tendon as well as the surrounding tissues in ultrasonography and magnetic resonance.

Comparing sonography and magnetic resonance imaging, they both failed to clearly depict the individual components of the common calcanean tendon throughout its overall course. In longitudinal/sagittal images, this was caused by the fact that the plane was set according to the long axis of the whole tendon, which does not correspond with the long axes of the particular tendons. This can be considered as an anatomical limitation for those imaging techniques, due to the extensive rotation of tendons in relation to their relatively short proximodistal dimensions. However, ultrasonography has an advantage of real time and dynamic examination, and allows for the manipulation of the probe placement over the superficial digital flexor tendon or the tendon of the medial head of the gastrocnemius. In transverse images, use of an ultrasound probe with a higher frequency range or higher magnetic field strength MRI unit might result in increased sensitivity allowing distinction of individual components of the common calcanean tendon in the rabbit. The echogenicity and signal intensity of the analyzed tendon observed in longitudinal/sagittal plane was generally comparable to that reported in the normal human calcaneal tendon (Bleakney and White 2005, Harris and Peduto 2006, Pierre-Jerome et al. 2010). The differences were the presence of the hypoechoic superficial component as detected in the sonographic examination and the linear areas of higher signal intensity as seen in magnetic resonance which represented the epitenon. Although, they might be compared to punctate or linear intermediate signal from normal fascicles occasionally detected in humans (Harris and Peduto 2006). In transverse planes echogenic/higher intensity punc- tate foci visible in our study are also reported in the human calcaneal tendon (Harris and Peduto 2006, Pierre-Jerome et al. 2010). Considering the assessment of the shape and margins of the common calcanean tendon in the rabbit, we conclude that magnetic resonance imaging provides better definition in transverse sections than ultrasonography, but both procedures assure good delineation of margins in longitudinal/sagittal plane.

The calcaneal tendon in humans is affected by a wide range of pathological conditions that may be examined in experimental research on animal models. Depending on specific pathology and its exact location, these disorders are characterized by different findings, involving various structures in diagnostic imaging techniques. We would like to discuss the usefulness of ultrasonography and magnetic resonance in the imaging of particular structures in a rabbit model in relation to human Achilles tendinopathies. It was found that the most commonly the calcaneal tendon rupture occurs ca. 2-6 cm from its insertion on the calcaneal tuber (Pierre-Jerome et al. 2010). However, atypical locations of tears also exist and proximal tears representing musculotendinous junction injury are more common than the distal ones (Pierre-Jerome et al. 2010). In the present study, musculotendinous junctions of the rabbit common calcanean tendon were well seen in both ultrasonographic and magnetic resonance images. The transition of muscle bellies to tendons was clear and evident. Insertional Achilles tendinopathy is associated with enthesophyte formation, bone erosion and calcification of the tendon at insertion site (McGonagle et al. 2008, Pierre-Jerome et al. 2010, van Dijk et al. 2011). In our rabbit model, magnetic resonance provided more detailed images of the region of the calcaneal insertion than sonography. In the present study, the paratenon was evident as a thin line surrounding the common calcanean tendon in both imaging methods, but ultrasonography also allowed for its visualisation caudally, whereas in magnetic resonance imaging it could not be distinguished from the subcutaneous tissue and the skin. Another important landmark is the pre-Achilles fat pad. Pathology of the calcaneal tendon or the talocalcaneal joint can result in obliteration and edema in the pre-Achilles fat pad visible in diagnostic imaging (Ly and Bui-Mansfield 2004, Pierre-Jerome et al. 2010). In our study, the pre-Achilles fat pad was detected with ultrasonography as a hypoechogenic or anechogenic structure with an irregular, blurred outline but magnetic resonance provided exact delineation of its shape and extent, both in sagittal and transverse planes. Finally, retrocalcaneal bursitis, i.e. inflammation of the bursa 
of calcaneal tendon, can be associated with a bone spur, bony enlargement or exostoses on the posterior aspect of the calcaneal tuber, biomechanical compression or can accompany insertional tendinopathy in humans (Pierre-Jerome et al. 2010, van Dijk et al. 2011). In our study, the bursa of the calcaneal tendon was not visible in ultrasonography or magnetic resonance probably due to its small size. In humans the normal bursa of calcaneal tendon seen on an MRI, measure less than $2 \mathrm{~mm}$ in antreoposterior dimension (Harris and Peduto 2006).

Statistical analysis confirmed that the craniocaudal diameter of the common calcanean tendon in the rabbit increases significantly with the growing distance from the calcaneal tuber. This relationship is finely imaged by the changes in shape observed in transverse sonographic and magnetic resonance scans. The lack of significant differences between measurements obtained with both imaging techniques proves their good visualisation of tendon margins in longitudinal/sagittal scans.

Magnetic resonance imaging and ultrasonography are both considered the diagnostic methods of choice regarding Achilles tendon assessment (Pierre-Jerome et al. 2010). They are based on different physical bases, and their advantages as well as their limitations are known (Shalabi et al. 2005, Nazarian 2008). However, there is still no consensus as to which of these modalities is more sensitive and reliable in evaluating the particular pathologies of the calcaneal tendon and predicting clinical outcome. In recent years, imaging techniques have developed dynamically, thus providing high-resolution sonography and high field strength MRI coupled with the introduction of doppler and contrast enhanced imaging as well as new sequences based on ultrashort echo times (Filho et al. 2009, Genovese et al. 2011, Del Buono et al. 2013). In our study we used a veterinary dedicated MRI unit and a standard linear-array orthopaedic ultrasound probe, both of which are easily accessible to a wide range of researchers.

In conclusion, the present study the low-field magnetic resonance provided more detailed images of the calcaneal insertion and the pre-Achilles fat pad than sonography did in a rabbit common calcanean tendon model. On the other hand, ultrasound assured better visualisation of the paratenon. They both failed to distinguish the individual tendons contributing to the common calcanean tendon as well as to image the bursa of the calcaneal tendon. We investigated only a baseline appearance of these structures. We conclude, that despite some limitations both magnetic resonance and ultrasonography are feasible, and should be considered complementary and not alternative imaging modalities in a rabbit model.

\section{Acknowledgements}

This research was supported by statutory research and development activity funds assigned to the Faculty of Veterinary Medicine, Wrocław University of Environmental and Life Sciences.

\section{References}

Benjamin M, Kaiser E, Milz S (2008) Structure-function relationships in tendons: a review. J Anat 212: 211-228.

Benjamin M, McGonagle D (2009) The enthesis organ concept and its relevance to the spondyloarthropathies. Adv Exp Med Biol 649: 57-70.

Bleakney RR, White LM (2005) Imaging of the Achilles tendon. Foot Ankle Clin 10: 239-254.

Del Buono A, Chan O, Maffulli N (2013) Achilles tendon: functional anatomy and novel emerging models of imaging classification. Int Orthop 37: 715-721.

Doherty GP, Koike Y, Uhthoff HK, Lecompte M, Trudel $\mathrm{G}$ (2006) Comparative anatomy of rabbit and human Achilles tendons with magnetic resonance and ultrasound imaging. Comp Med 56: 68-74.

Doral MN, Alam M, Bozkurt M, Turhan E, Atay OA, Donmez G, Maffulli N (2010) Functional anatomy of the Achilles tendon. Knee Surg Sports Traumatol Arthrosc 18: 638-643.

Filho GH, Du J, Pak BC, Statum S, Znamorowski R, Haghighi P, Bydder G, Chung CB (2009) Quantitative characterization of the Achilles tendon in cadaveric specimens: $\mathrm{T} 1$ and $\mathrm{T} 2 *$ measurements using ultrashort-TE MRI at 3T. AJR Am J Roentgenol 192: $117-124$

Genovese E, Ronga M, Recaldini C, Fontana F, Callegari L, Maffulli N, Fugazzola C (2011) Analysis of Achilles tendon vascularity with second-generation contrast-enhanced ultrasound. J Clin Ultrasound 39: 141-145.

Harris CA, Peduto AJ (2006) Achilles tendon imaging. Australas Radiol 50: 513-525.

Hast MW, Zuskov A, Soslowsky LJ (2014) The role of animal models in tendon research. Bone Joint Res 3: 193-202.

Kader D, Saxena A, Movin T, Maffulli N (2002) Achilles tendinopathy: some aspects of basic science and clinical management. Br J Sports Med 36: 239-249.

Kamel M, Eid H, Mansour R (2003) Ultrasound detection of heel enthesitis: a comparison with magnetic resonance imaging. J Rheumatol 30: 774-778.

Leung JL, Griffith JF (2008) Sonography of chronic Achilles tendinopathy: a case-control study. J Clin Ultrasound 36: 27-32.

Ly JQ, Bui-Mansfield LT (2004) Anatomy of and abnormalities associated with Kager's fat pad. AJR Am J Roentgenol 182: 147-154.

McGonagle D, Wakefield RJ, Tan AL, D'Agostino MA, Toumi H, Hayashi K, Emery P, Benjamin M (2008) Distinct topography of erosion and new bone formation in Achilles tendon enthesitis: implications for understanding the link between inflammation and bone formation in spondylarthritis. Arthritis Rheum 58: 2694-2699. 
Moshiri A, Oryan A (2011) Structural and functional modulation of early healing of full-thickness superficial digital flexor tendon rupture in rabbits by repeated subcutaneous administration of exogenous human recombinant basic fibroblast growth factor. J Foot Ankle Surg 50: 654-662.

Nazarian LN (2008) The top 10 reasons musculoskeletal sonography is an important complementary or alternative technique to MRI. AJR Am J Roentgenol 190: 1621-1626.

Oryan A, Moshiri A (2011) A long term study on the role of exogenous human recombinant basic fibroblast growth factor on the superficial digital flexor tendon healing in rabbits. J Musculoskelet Neuronal Interact 11: 185-195.

Oryan A, Moshiri A, Meimandi Parizi AH, Raayat Jahromi A (2012) Repeated administration of exogenous sodium-hyaluronate improved tendon healing in an in vivo transection model. J Tissue Viability 21: 88-102.

Pierre-Jerome C, Moncayo V, Terk MR (2010) MRI of the Achilles tendon: a comprehensive review of the anatomy, biomechanics, and imaging of overuse tendinopathies. Acta Radiol 51: 438-454.

Shalabi A, Movin T, Kristoffersen-Wiberg M, Aspelin P, Svensson L (2005) Reliability in the assessment of tendon volume and intratendinous signal of the Achilles tendon on MRI: a methodological description. Knee Surg Sports Traumatol Arthrosc 13: 492-498.

Shapiro E, Grande D, Drakos M (2015) Biologics in Achilles tendon healing and repair: a review. Curr Rev Musculoskelet Med 8: 9-17.

Skalec A, Janeczek M, Janus I, Chrószcz A, Henklewski $\mathrm{R}$ (2016) Rabbit common calcanean tendon as an animal model: ultrasonographic anatomy and morphometry. Folia Morphol 75: 93-100.

Szaro P, Witkowski G, Śmigielski R, Krajewski P, Ciszek B (2009) Fascicles of the adult human Achilles tendon - an anatomical study. Ann Anat 191: 586-593.

Theobald P, Bydder G, Dent C, Nokes L, Pugh N, Benjamin $M$ (2006) The functional anatomy of Kager's fat pad in relation to retrocalcaneal problems and other hindfoot disorders. J Anat 208: 91-97.

Thomopoulos S, Parks WC, Rifkin DB, Derwin KA (2015) Mechanisms of tendon injury and repair. J Orthop Res 33: 832-839.

Trudel G, Koike Y, Ramachandran N, Doherty G, Dinh L, Lecompte M, Uhthoff HK (2007) Mechanical alterations of rabbit Achilles' tendon after immobilization correlate with bone mineral density but not with magnetic resonance or ultrasound imaging. Arch Phys Med Rehabil 88: $1720-1726$.

van der Made $\mathrm{AD}$, Maas M, Beenen LF, Oostra RJ, Kerkhoffs GM (2013) Postmortem imaging exposed: an aid in MR imaging of musculoskeletal structures. Skeletal Radiol 42: 467-472.

van Dijk CN, van Sterkenburg MN, Wiegerinck JI, Karlsson J, Maffulli N (2011) Terminology for Achilles tendon related disorders. Knee Surg Sports Traumatol Arthrosc 19: 835-841.

Young RG, Butler DL, Weber W, Caplan AI, Gordon SL, Fink DJ (1998) Use of mesenchymal stem cells in a collagen matrix for Achilles tendon repair. J Orthop Res 16: 406-413. 